\title{
Reviewers for the Journal of the American Board of Family Medicine in 2006
}

External peer reviewers provide a vital service to the $7 A B F M$ and to the family medicine specialty. By donating their time and expertise to reading manuscripts and writing critiques, reviewers assist authors to improve their manuscripts and raise the quality of the work published in the $7 A B F M$. Journal peer reviewing also offers a number of benefits for researchers. This form of professional socialization is an avenue for the reviewer to improve his or her own critical appraisal and writing skills.

Reviewers occasionally ask for clarification about the biomedical publishing process. To assist in the professional development of our reviewers, the $7 A B F M$ provides some resources in the Peer Reviewer area of the $\mathcal{F} A B F M$ website. The article "The Peer Review Process of the $\mathcal{F} A B F M$," available at http:// www.jabfm.org/cgi/content/full/19/2/209, describes an overview of our procedures related to peer review and editorial decision making. Specific information about what we expect from our reviewers is outlined in the article "Peer Reviewing for the $7 A B F M$ : What Does it Take?," available at http://www.jabfm.org/cgi/content/full/19/6/643, which provides additional details about our peer-review policies and procedures, presents the ethical issues in peer review, and discusses the qualities of a good peer review.

Authors and researchers are invited to enlist as a peer reviewer for the $\mathcal{F} A B F M$ (see the peer reviewer registration form on the website at www.jabfm.org). We aim to secure a specialist and a generalist for each reviewed manuscript to provide a broad and scholarly critique to assist authors in disseminating their work.

In 2006, the 352 individuals named below wrote reviews for the $7 A B F M$. If you provided a peer review to the $\mathcal{A} A B F M$ in 2006 and your name is not listed, please let us know.

Wael A. Aboughali

Louise S. Acheson

David C. Agerter

Sanjiv Ahluwalia

Erin J. Aiello

James E. Aikens

Grace A. Alfonsi

William A. Alto

Gail M. Amundson

Sven O. Andersson

Emmanuel Andres

Thomas A. Arcury

Bengt Arnetz

Cheryl B. Aspy

Latifi A. Asrar

John Bachman

Beth Barnet
Donald Barr

Kirsten Barrett

Henry C. Barry

Kay A. Bauman

Max Bayard

John W. Beasley

Lorne A. Becker

Howard B. Beckman

Marvin Moe Bell

Ian M. Bennett

Alfred O. Berg

Ethan Berke

Jeralyn A. Bernier

Juliann Binienda

Helen J. Binns

Sean M. Blaine

Barry Bleidt
Carol E. Blenning

Richard D. Blondell

B. Wayne Blount

Thomas Bodenheimer

Tanja Britton

Fredrik F. Broekhuizen

David R. Brown

Sharon A. Brown

Sean T. Bryan

Frank Buntinx

Sandra K. Burge

Marguerite Burns

Javed Butler

Doug Campos-Outcalt

Peter J. Carek

Nancy Carter

Frederick M. Chen 
Jack A. Clark

Richard D. Clover

Vilma Cokkinides

Priscilla K. Coleman

Jennie Connor

Richard A. Cooper

Tamera Coyne-Beasley

Monica K. Crane

Paul F. Crawford III

Jesse C. Crosson

Michael A. Crouch

Brian K. Crownover

Richard W. Crummer

Larry Culpepper

Doyle M. Cummings

R. Whit Curry Jr.

Craig W. Czarsty

Timothy P. Daaleman

Robin M. Daly

Christian Daughton

Alan K. David

Matthew Davis

Martin G. Dawes

Darwin Deen

Frank deGruy

Mark J. DeHaven

Cristina Demian-Popescu

Judith D. DePue

Vanessa Diaz

Lori M. Dickerson

L. Miriam Dickinson

Lisabeth F. DiLalla

Mark P. Doescher

Tanya Dougherty

David Doukas

Margaret M. Eberl

Jennifer J. Eddy
Kristen Ehresmann

Nancy C. Elder

William G. Elder Jr.

Eva K. Ellegard

R. Curtis Ellison

John W. Ely

William D. Engle

John W. Epling Jr.

Luca D. Esposti

Bernard G. Ewigman

Wei Li Fang

Deb Feldman-Stewart

Maria-Luz Fernandez

Daron G. Ferris

Judith A. Fifield

Edgar Figueroa

Alexander G. Fiks

Kenneth S. Fink

Kevin Fiscella

Sue Flocke

Kory Floyd

Colleen T. Fogarty

Samuel N. Forjuoh

Stuart Forman

Chester H. Fox

Kimberley K. Fox

Peter Franks

Linda M. French

John J. Frey III

Keith A. Frey

Ivar L. Frithsen

Sim S. Galazka

James M. Galliher

Mauro Gallitelli

Joseph J. Gallo

David Garr

David L. Gaspar
Neeta V. Gautam

Suzanne Gehl

Barbara Geller

Thomas P. Gessner

Valerie J. Gilchrist

James M. Gill

Dwenda K. Gjerdingen

Rise B. Goldstein

Wanda C. Gonsalves

Jodi M. Gonzalez

Roland M. Grad

Ken Grauer

Lisa Graves

Beverly B. Green

Larry A. Green

Michael E. Green

Greg Greenberg

Kim Griswold

Joseph B. Guarnaccia

Karen Gunning

Leonard J. Haas

Daniel E. Hall

Gloria M. Halverson

Laura B. Hansen

Arthur J. Hartz

Kelly Haskard

Robert L. Hatch

Sarah Hawley

Diane G. Heatley

Christopher J. Hebert

Robin Helm

Nikhil Hemady

Vincent W. Hevern

David E. Hildebrandt

Robert S. Holzman

Eugene Hong

Margaret S. Houston 
William J. Hueston

David Hyman

Carol L. Ireson

Jamal Islam

Donna B. Jeffe

Masahito Jimbo

Chris W. Johnson

Thomas A. Johnson Jr.

Eva Kahana

Stephanie Y. Kao

Ben-Tzion Karsh

Neil S. Kaye

Robert D. Keeley

Omar A. Khan

Shersten Killip

Dana E. King

Anita Y. Kinney

Kyle J. Kircher

Jeffrey T. Kirchner

Howard S. Kirshner

Joseph C. Konen

Colin P. Kopes-Kerr

Claudia A. Kozinetz

Katriina Kukkonen-Harjula

Grace M. Kuo

Anton J. Kuzel

Forrest Lang

Cristina Lara-Castro

Frank Lawler

Beverley A. Lawton

Rebecca Lawton

Lawrence M. Leeman

Bruce Leff

Carmen W. Liang

Cara Liday

Charles F.S. Locke

Jason A. Logan
Everett E. Logue

Lucy W. Loomis

Karl Lorenz

David P. Losh

Jeffrey P. Louie

Bing Lu

Jun Ma

Paula K. Maasilta

Ann Macaulay

Caroline A. Macera

Michael K. Magill

Megan R. Mahoney

Cara Marshall

Catherine McCarthy

Susan H. McDaniel

Helen E. McIlvain

James R. McKay

Helen E. Meador

Joel H. Merenstein

Daniel L. Meyer

Donald B. Middleton

John E. Midtling

Fred Miser

James E. Mitchell

James W. Mold

Alain J. Montegut

Karen Muchowski

Joseph M. Murley

Zsolt J. Nagykaldi

Sachidanandan Naidu

Rajasree J. Nair

Laeth S. Nasir

Donald E. Nease Jr.

Dorothy A. Nelson

Warren P. Newton

Giang T. Nguyen

Thomas E. Norris
Mary Patricia Nowalk

Cathleen O'Farrell

Steven M. Ornstein

Judith A. Owen

Wilson D. Pace

Cristy Page

Heather Paladine

Donald Pathman

Katherine L. Patterson Neely

Valory N. Pavlik

Kevin A. Pearce

William S. Pearson

Marco Pennazio

Robert A. Peterfreund

Michael E. Pichichero

Joesph M. Pierre

Marty S. Player

Charles D. Ponte

John H. Porcerelli

M. C. Post

Bruce Prenner

Wayne Putnam

Howard K. Rabinowitz

Kalyanakrishnan Ramakrishnan

Joanne L. Reeve

Michael D. Reis

Peter L. Reynolds

Alfred A. Rimm

Peter A. Rives

W. David Robinson

John C. Rogers

Roger A. Rosenblatt

Marjorie Sue Rosenthal

Michael P. Rosenthal

Steven E. Roskos

Michael G. Ross

Mack T. Ruffin IV 
Terry S. Ruhl

Kaveh Safavi

Ramesh Saxena

Candice L. Schachter

Maryjean Schenk

Joseph E. Scherger

Sarina Schrager

Kendra L. Schwartz

Thomas L. Schwenk

Ian Scott

Peter A. Selwyn

Richard K. Severson

Karen Shashok

Eric K. Shaw

Neil S. Silverman

Ajai Singh

Peter C. Smith

Kevin Sneed

Leif I. Solberg

Claire Somerville

Stuart M. Speedie

Susan E. Spengler

Theodore Speroff

Jeffrey P. Staab

Jamie Stang

Phyllis K. Stein

Rosemary A. Stevens
Gregg D. Stoner

Mark L. Stovak

Steven D. Stovitz

Henrik Stovring

Joseph Straton

Scott M. Strayer

Jeremy Sugarman

Andrew L. Sussman

John E. Sutherland

David E. Swee

Alfred F. Tallia

Deanna E. Telner

John E. Tetzlaff

Siwan Thomas-Gibson

Dixie L. Thompson

Tom Tomlinson

Peter P. Toth

John M. Tovar

Suzanne Trupin

Keiichi Uchida

Matthew E. Ulven

Monte C. Uyemura

Daniel J. Van Durme

Peter Vasilenko

Daniel C. Vinson

Michael Von Korff

Gordon Walbroehl
Shari R. Waldstein

Eric Wall

Judith M.E. Walsh

Marilyn T. Wayland

Ashley Wazana

Ganesa Wegienka

Frances K. Wen

Johan Wens

Randy K. Wexler

Barbara E. Wildhaber

Thad Wilkins

Margaret L. Williams

Thad E. Wilson

Robin Winter

William M. Woodhouse

W. Michael Woods

Susan Woolhouse

Xiao $\mathrm{Wu}$

Bektas Murat Yalcin

Rosalie F. Young

Atif Zafar

Michael Zemel

Therese Zink

Adam J. Zolotor

Steven Zweig 